\title{
SOLUTIONS OF THE LANDAU-VLASOV EQUATION IN NUCLEAR PHYSICS
}

\author{
B. REMAUD ${ }^{* *}$, F. SEBILLE*, C. GREGOIRE** and L. VINET ${ }^{* * *}$ \\ "Institut de physique, Université de Nantes, 2, rue de la \\ Houssinière, F-44072 Nantes Cedex, France \\ * IRESTE, 3, rue du Maréchal Joffre, Université de Nantes, \\ F-44041 Nantes Cedex 01, France \\ ** "GANIL, BP 5027, F-14021 Caen Cedex, France
}

Résume: Nous discutons les propriétés des solutions de l'équation de Vlasov obtenues par projection sur une base d'etats coherents. Elles vérifient des conditions de stationnarité et permettent de décrire la diffusivité moyenne de l'espace de phase nucleaire et de reproduire les caractéristiques moyennes des noyaux. Les methodes d'échantillonnage et leurs effets sur la dynamique sont discutés pour l'étude des réactions nucléaires aux énergies intermédiaires. Enfin, l'utilisation de la force non locale de Gogny est directe ce qui donne l'opportunite de l'utiliser en dynamique nucléaire.

Abstract: The properties of Vlasov equation solutions obtained by projection on coherent state basis are discussed. Such solutions satisfy stationarity conditions and satisfactorily describe the average diffusivity of nuclear phase space and reproduce the bulk properties of nuclei. Sampling methods and their effects on dynamics are discussed for the study of heavy ion reactions at intermediate energies. The non-local Gogny force is easily computable on this basis which allows to use it for dynamical nuclear studies.

\section{I-INTRODUCTION.}

The new experimental data obtained in the intermediate energy range (10 to $100 \mathrm{~A}-\mathrm{MeV}$ ) have brought forward the Vlasov equation (and its associates the Landau-Vlasov'(1) or V.U.U. equation (2)) in the field of nuclear studies. It gives the time evolution of the nucleus one-body phase space under the influence of the nuclear mean-field :

$$
\mathrm{D}_{\mathrm{t}} \mathrm{f} \equiv \frac{\partial \mathrm{f}}{\partial \mathrm{t}}+[\mathrm{f}, \mathrm{H}]=0
$$

$f=f(\vec{r}, \vec{p} ; t)$ is the one-body density distribution; the Poisson brackets (\} are defined as usual from the Hamiltonian $H(\vec{r}, \vec{p})$ :

$$
[\mathrm{f}, \mathrm{H}]=\overrightarrow{\nabla_{\mathbf{r}} \mathrm{f}} \cdot \overrightarrow{\nabla_{p} \mathrm{H}}-\overrightarrow{\nabla_{p} \mathrm{f}} \cdot \overrightarrow{\nabla_{r} \mathrm{H}}
$$

We shall not discuss here the virtues and drawbacks of such an equation which constitute a lively subject of discussion between theoreticians. We shall take it as it is : a semi-classical approximation to more soph isticated self-consistent quantum evolution 
equations ; in particular it can be found as the $h \rightarrow 0$ limit of TDHF equation (3). Then $f(\vec{r}$, $\vec{p})$ is a smoothed, positive approximation to the exact Wigner-transform of the one body nuclear density matrix.

The appealing features of Vlasov equation in nuclear physics are threefold i) since it is positive, $f(\vec{r}, \vec{p} ; t)$ can be interpreted as a probability distribution of an assembly of (fictious) pseudo-particles ; ii) its semi-classical character provides easier interpretations of its properties; iii) it can be coupled to a collision term to take into account the residual interaction. One of the unpleasant features of the Vlasov equation lies in the fact that it is not easier to solve than the TDHF equation from which it can be derived. However, the Vlasov equation turns out to be a - still unchallenged - method to studying the interplay between one - and two-body interactions in heavy-ion collisions.

In this paper, we shall discuss two questions which are connected with the use of Vlasov equation in nuclear physics i) how can we define its initial conditions ii) what are the methods to solve it taking advantage of its considerable redundancy. The first point is of major importance at lower energies since the close-to-equilibrium properties cannot be safely studied unless one is able to provide descriptions of nuclear ground states which are stationary solutions of Vlasov equation. The second one is essential to the possibility of making simulations of real nuclear reactions.

The next section presents the basis that we use ; the third section gives some results for nuclear statics. Dynamics of nuclear reactions is discussed in section 4

\section{II - STATIC BASIS OF THE PHASE SPACE.}

The coherent states are gaussian wave packets labelled by a continuous complex parameter $\mid Z>$, connected with the position of their center $\left(x_{0}, p_{0}\right)$; we firstly discuss here the one-dimension case since the extension of the formalism to three dimensions is straightforward. The coherent state properties are well known (see ref. 4 for a review). Let us briefly recall that i) according to the metric $d \mu(z)=d x_{O} d p_{O} / h_{\text {, }}$ they obey the closure relationship: $\left.\quad \int d \mu(z) z\right\rangle\langle z|=\hat{1}$

ii) they form an overcomplete basis of the Hilbert space ; i.e. one can develop any density operator as $\quad \hat{\mathrm{p}}=\int \mathrm{d} \mu(z) d \mu\left(z^{\prime}\right) p_{z z^{\prime}}\left|z><z^{\prime}\right|$

which determines a mapping of the quantum phase space onto the space spanned by the continuous parameters $z$.

The coherent states are quantum objects, particularly well fitted to the semi-classical approximations. The Wigner transform of $\langle\mathrm{x} \mid \mathrm{Z}\rangle$ is :

$$
\mathrm{d}\left(\mathrm{x}-\mathrm{x}_{0}, \mathrm{p}-\mathrm{p}_{0} ; \Delta \mathrm{x}, \Delta \mathrm{p}\right)=(2 \pi \Delta \mathrm{x} \Delta \mathrm{p})^{-1} \exp \left[-\frac{1}{2}\left(\frac{\mathrm{p}-\mathrm{p}_{0}}{\Delta \mathrm{p}}\right)^{2}-\frac{1}{2}\left(\frac{\mathrm{x}-\mathrm{x}_{0}}{\Delta \mathrm{x}}\right)^{2}\right)^{2}
$$

Although they originates from minimum uncertainty wave packets $(\Delta \mathrm{p} \Delta \mathrm{x}$ $=h / 2$ ) ; one can use reduced coherent states (i.e. $\hbar \rightarrow 0$ ) while preserving the above properties 2.1 and 2.2. In this case, they cannot be interpreted as wave packets of real particles but as basis elements of the phase space (they are often called "quasi particles") 
which cannot be interpreted independently each from others. Notice that we recover the "classical quasi particles" $\delta\left(x-x_{0}, p-p_{O}\right)$ if $\hbar=0$.

The projection of a given phase space distribution then reads

$$
\mathrm{f}(\mathrm{x}, \mathrm{p})=\mathrm{W}(\mathrm{x}, \mathrm{p}) * \mathrm{~g}(\mathrm{x}, \mathrm{p} ; \Delta \mathrm{x}, \Delta \mathrm{p}) ;
$$

it is a folding product of the coherent states basis on a weight function w(x,p)

$$
\begin{aligned}
f(x, p)= & (4 \pi \hbar \Delta x \Delta p)^{-1} \int d x_{0} d p_{0} w\left(x_{0}, p_{0}\right) g\left(x-x_{0}, p-p_{0}: \Delta x, \Delta p\right) \quad \text { (2.5) } \\
& \text { In principle, one could deduce such expressions from } p_{Z Z} \text { in eq. } 2.2 .
\end{aligned}
$$

However, in this case the resulting $f(x, p)$ is not fitted to our goals since it keeps track of all quantum effects (such as shell effects) and presents wide oscillations. The general method to deduce a smooth approximation $f(x, p)$ from eq. 2.4 is still to be performed. We have used a very simple ansatz (1) guided by the Thomas-Fermi limit that we must recover at the $h \rightarrow 0$ limit and by quantization methods of semi classical orbits (5).

$$
f(x, p)=\theta\left(\varepsilon_{F}-\bar{H}(x, p)\right) * d(x, p, ; \Delta x, \Delta p)
$$

The symbole $\overline{\mathrm{H}}$ indicates that one takes the expectation value of the classical hamiltonian on the coherent state, which is imposed by energy-conservation conditions in dynamical equations (see below). Eq. 2.6 extends the existence domain of $f(x, p)$ beyond the classical allowed region-while not inducing any coupling to the continuum. As in most smoothing procedures, we are left with the choice of the couple $(\Delta x, \Delta p)$ which is fixed by requiring that the average diffusivity of the real phase space is reproduced. We notice that in eq. 2.5. the step function plays the role of an occupation number for a continuous set of semi-classical trajectories. In fig. 1, one can see the comparison between eq. 2.6 for fermions in a harmonic field and exact ar smoothed quantum results.

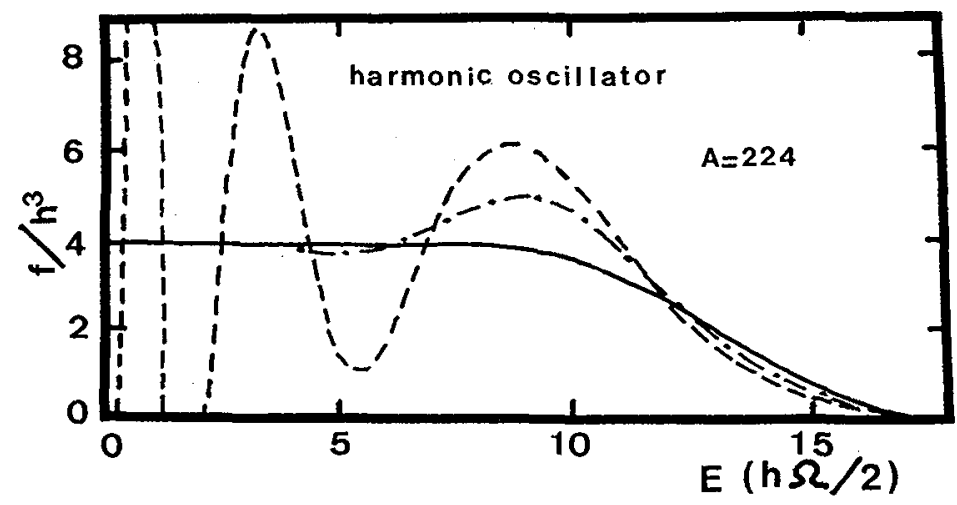

Fig.1 : Phase density distributions in $(2 \pi \hbar)^{3}$ units for 224 fermions in a harmonic well The exact (dashed curve) and smoothed (dashed and dotted curve) solutions are from ref.10. Our result is given by the full line.

\section{III - STATIC DESCRIPTION OF NUCLEI.}

The phase space distribution of a cold nucleus is given by a direct extension of eq. 2.6: 


$$
f(\vec{r}, \vec{p})=\theta(\varepsilon F-\vec{H}(\vec{r}, \vec{p})) * d(\vec{r}, \vec{p} ; \Delta r, \Delta p)
$$

where we assume isotropy in the $\vec{r}$ and in the $\vec{p}$ directions. The Fermi energy $\varepsilon_{F}$ is self-consistently deduced by constraining the norm of $f(\vec{r}, \vec{p})$ to the number of nucleons Till now, calculations have been performed with simple Skyrme effective interactions :

$$
U(\rho(\vec{r}))=a p(\vec{r})+b p^{v+1}(\vec{r}) \quad 0<v<1
$$

which are easily combined with eq. 3.1 , since the spatial density $\rho(\vec{r})$ is :

$$
\rho(\vec{r})=\int f(\vec{r}, \vec{p}) d \vec{p}=f_{T F}(\vec{r}) * d(\vec{r} ; \Delta r)
$$

i.e. the Thomas-Fermi density (taking into account the zero-point energy) folded with a gaussian.

However, the structure of eq. 3.1 is particularly well fitted to the use of the Gogny effective interaction (6) which is non-local and allows good descriptions of ground state as well as fissionning nuclei. In the phase space notation, the one particle potential reads for $\mathrm{N}=\mathrm{Z}$ nuclei ( 7 )

$$
\begin{aligned}
\mathrm{V}_{\mathrm{HF}}(\overrightarrow{\mathrm{r}})= & \frac{7}{8} \mathrm{t}_{3} \rho^{4 / 3}(\overrightarrow{\mathrm{r}})+\sum_{\mathrm{i}=1}^{2} A_{\mathrm{i}} \rho(\overrightarrow{\mathrm{r}}) * \mathrm{~d}\left(\overrightarrow{\mathrm{r}}, \mu_{\mathrm{i}} / \sqrt{2}\right) \\
& +\sum_{\mathrm{i}=1}^{2} \mathcal{B}_{\mathrm{i}} \mathrm{f}(\overrightarrow{\mathrm{r}}, \overrightarrow{\mathrm{p}}) * \mathrm{~d}\left(\overrightarrow{\mathrm{p}} ; \sqrt{2 \hbar / \mu_{\mathrm{i}}}\right)
\end{aligned}
$$

The widths $\mu_{1}$ and depths $\mathscr{A}_{1}, B_{1}$ are deduced from the D1 parametrization of the Gogny force. Since folding products between gaussians are straightforward

$$
g\left(x, \alpha^{1 / 2}\right) * g\left(x, \beta^{1 / 2}\right)=g\left(x,(\alpha \beta)^{1 / 2}\right)
$$

the Gogny force turns out to be handy for semi-classical static and dynamic nuclear study ; it provides an effective way of studying the effects of non-locality and/or velocity dependence on observables in heavy-ion reactins. In fig. 2 , we show the nuclear profile obtained for a ${ }^{40} \mathrm{Ca}$ nucleus.

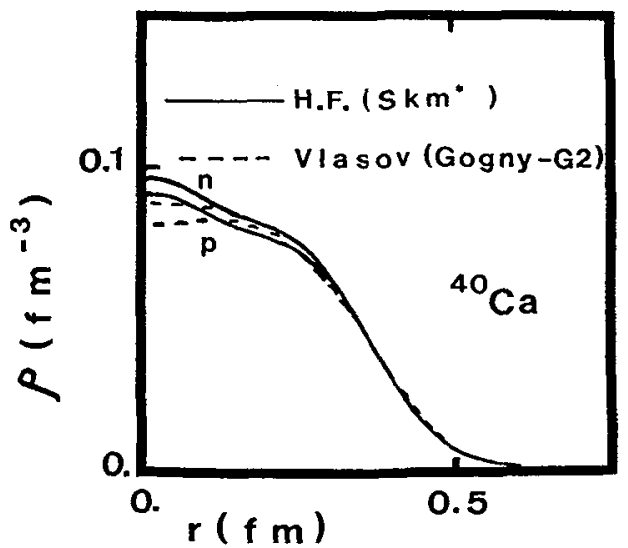

Fig.2 : Density profiles for protons, neutrons in $a^{40} \mathrm{Ca}$ nucleus. We compare the results of Hartree-Fock with a Skyrme interaction and our calculation with a cogny interaction. 


\section{IV - NUCLEAR DYNAMICS.}

The main objective of the above considerations on nuclear statics is to get good initial conditions of the Vlasov (or V.U.U.) equation. If any functional of energy is solution of the static (time independent) Vlasov equation, one has to demonstrate that it is stationary before using it in dynamical calculations, i.e. that all the observables are time-independent at least on a time-scale lager than the average duration of the nuclear reaction.

Solutions of eq. (1.1) having the form of eq. (3.1) are given provided that i) $\mathrm{H}$ is a constant of motion and ii) the coherent states are solutions of the Vlasov equation. The first condition :

$$
\frac{\mathrm{d}}{\mathrm{dt}} \overline{\mathrm{H}}(\overrightarrow{\mathrm{r}}, \overrightarrow{\mathrm{p}})=0
$$

is exactly fulfilled if the gaussian centers follow the Ehrenfest equations :

$$
\begin{aligned}
& \frac{d \vec{r}_{0}}{d t}=\bar{\nabla} p_{0} H\left(\vec{r}_{0}, \vec{p}_{0}\right) \\
& \frac{d \vec{p}_{0}}{d t}=-\bar{r}_{r_{0}} H\left(\vec{r}_{0}, \vec{p}_{0}\right)
\end{aligned}
$$

The second candition is fulfilled in the sense that eqs. (4.2) insure the minimal value of the action associated with the coherent state propagation in general potential. Our semi-classical approximation then amounts to project the exact phase space on the class of solutions given by a swarm of coherent states moving in an effective field $\overline{\mathrm{H}}$. The stationarity is guaranteed since only the lower energy orbits are fully occupied. These properties have been checked with a very high degree of accuracy : absolute fluctuations of less than $1 \%$ of the total energy, the rms radius and quadrupole moments which are the low energy modes immediately excited by small deviations from the ground state.

However, the phase space occupied by a nucleus is very large when compared to the actual extension of a coherent states, the ratio scales roughly as

$$
\tau=(R / \Delta r)^{3}\left(p_{F} / \Delta p\right)^{3}
$$

where $R$ and $p_{F}$ are respectively the radius and the Fermi momentum of the nucleus For nuclei with $A \gg 1, T$ is of the order of one thousand per nucleon (in our calculations. typical values for $\Delta r$ and $\Delta p$ are respectively 0.6 fermi and 0.15 fermi $^{-1}$ ). Real calculations can be performed in two cases $i)$ when symmetries reduce the dimensionality of the problem, one can track the whole phase space (see ref 8 for spherical symmetries). ii) For real 3-D problems, one has to reduce the complexity by a Monte-Carlo sampling of the step function in eq. 3.1 :

$$
f(\vec{r}, \vec{p}) \approx \sum_{i=1}^{N} w_{i} d\left(\vec{r}-\vec{r}_{i} ; \vec{p}-\vec{p}_{i} ; \Delta r, \Delta p\right)
$$


The number $N$ is evidently a critical parameter, numerical tests show that complete stability is obtained as soon as $N \geqslant 50 * A$ which is still large but much less than eq (4.3) would let expect.

We shall not show here the variety of results that one can extract from such calculations which go from the collective mode excitation of cold or heated nuclei (8) to the intermediate energy reaction mechanism (9). These results show the ability of the formalism to follow the time evolution as well of relatively low energy modes as of reactions where considerable energy and momentum transfers occur.

In fig. 3 , we show calculations for the ${ }^{12} \mathrm{C}+{ }^{12} \mathrm{C}$ reaction at $84^{*} \mathrm{~A} \mathrm{MeV}$ with the Gogny interaction ; it is compared with calculations made with a simple local Skyrme interaction. In this central collision, the bulk behavior looks similar, but we must remark that the final relative velocities are different, this effect can be tracked back to the potential energy evolution which exhibits strong variations at the beginning of the reaction due to the non-local character of the interaction. This preliminary result shows the open field of research that one can expect from the Landau-Vlasov equation used with such sophisticated interactions ${ }^{11)}$

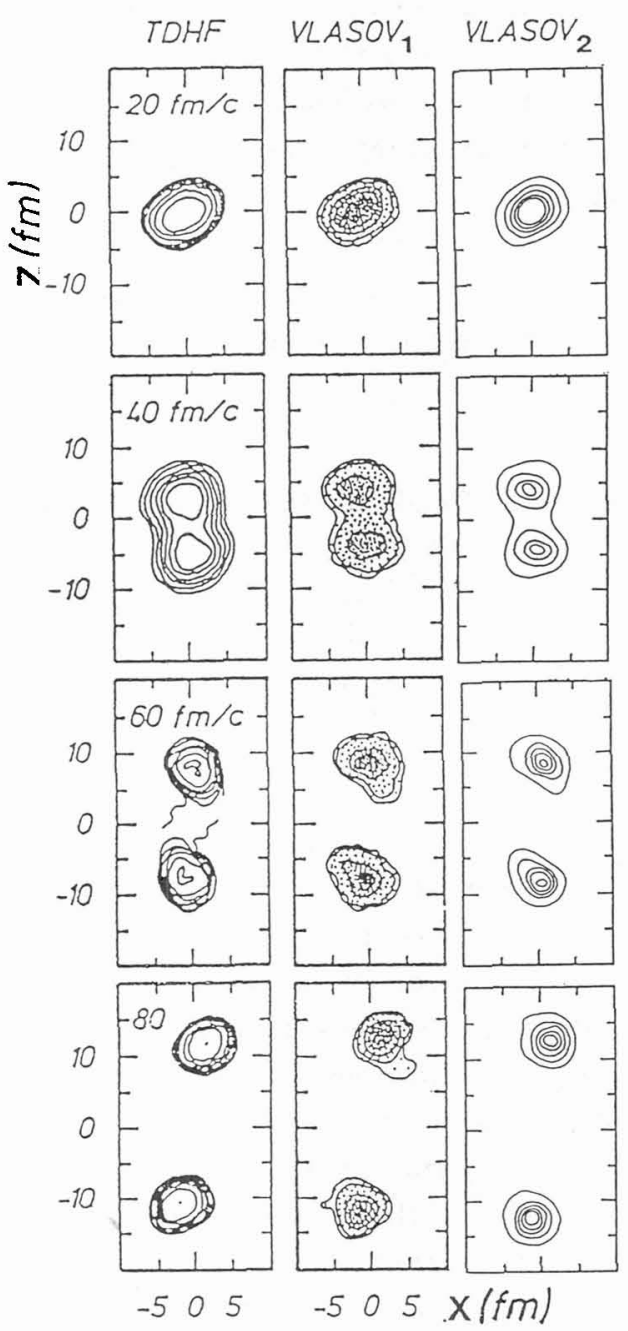

Fig.3 : Projected nuclear densities on the reaction plane for the ${ }^{12} \mathrm{C}+{ }^{12} \mathrm{C}\left(84^{*} \mathrm{~A} \mathrm{MeV}\right)$ reaction the impact parameter is $2.5 \mathrm{fm}$. The results labelled as TDHF and 'VLASOV 1 are found with a SKYRME force, VLASOV 2 gives the results with the GOGNY interaction.

\section{V-CONCLUSIONS.}

We have discussed a sheme to solve the Vlasov (Landau-Vlasov and V.U.U) equations in the context of nuclear physics. We have shown that gaussian (coherent) states are particularly well-fitted moving bases on to which one can project the dynamic solutions. The finite-size character of the gaussians are essential to the correct description 
of the bulk static properties of nuclei and then to the study of low-energy collective modes of nuclei. Taking use of the gaussian form factors of the non-local Gogny interaction. we have shown that it can be used in dynamical studies; this interaction has been very successful in the study of static nuclear properties and of fission modes, our formalism provides a unique way of using it in the study of collective mode excitations and reaction mechanisms.

The authors thank M. PI, Y. RAFFRAY, P. SCHUCF and E. SURAUD for their collaborations.

\section{REFERENCES}

1) C. GREGOIRE, B. REMAUD, F. SEBILLE, L. VINET, Nucl. Phys. A447 (1985) 55c and Nucl. Phys. A465 (1987) 317.

2) J. AICHELIN, H. STOCKER, Phys. Lett. 163B (1985) 59 and references therein.

3) P. CARRUTHERS. F. ZACHARIASEN, Rev. Mod. Phys. 55 (1983) 245.

4) R.J. GLAUBER, in Lecture in Theoretical Physics, Vol 1, ed. W.E. Brittin (Interscience. New York, 1959) 315.

5) E.J. HELLER, J. Chem. Phys. 75 (1981) 2923.

6) J. DECHARGE, D. GOGNY, Phys. Rev. C21 (1980) 1568.

7) A. AYASHI, P. SCHUCK (private communication)

8) L. VINET, C. GREGOIRE, P. SCHUCK, B. REMAUD, F. SEBILLE, Nucl. Phys (in Press).

9) C. GREGOIRE, This Conference and references therein:

10) M. PRAKASH, S. SHLOMO, V.M. KOLOMIETZ, Nucl. Phys. A370 (1981) 30.

11) F. SEBILLE et al to be published. 